\title{
Change Detection and Classification Using High Resolution SAR Interferometry
}

\author{
Azzedine Bouaraba, Nada Milisavljević, \\ Marc Acheroy and Damien Closson \\ Additional information is available at the end of the chapter \\ http://dx.doi.org/10.5772/57246
}

\section{Introduction}

Change detection with Synthetic Aperture Radar (SAR) images involves often a pair of co-registered images acquired over the same area at different times [1]. To identify changes, different methods are commonly applied. These methods differ with respect to the parameters that are used to indicate changes. Since SAR data contain amplitude and phase information, both parameters can be used as change indicators [2,3].

Incoherent change detection identifies changes by comparing sample estimates of the mean backscatter power taken from the repeat pass image pair [4, 5]. The Coherent Change Detection (CCD) technique uses the coherence of a SAR image pair to quantify changes in the observed amplitude and phase of the image pixels. As the Interferometric SAR (InSAR) phase is sensitive to changes in the spatial distribution of scatterers within a resolution cell, the CCD technique that generally detects the low-coherence areas as ground changes $[6,7]$ has the potential to detect centimeter changes that may remain undetected using only SAR intensity images (incoherent detection) [4, 8].

With the recent incoming of satellite constellations, delivering high-resolution SAR images, it becomes possible to detect surface changes with fine spatial details and with a short revisiting time. This aspect makes the CCD technique ideal for use in military and scientific applications such as border security and environmental monitoring. All these reasons lead to the development of new methods that enhance the change detection performance $[4,8,9]$. However, two main difficulties must be overcome in order to improve the analysis of CCD results.

The first difficulty concerns the InSAR coherence misestimation. Indeed, the sample coherence estimator is biased, especially for low-coherence values [7, 10]. In addition to the presence of speckle in SAR data [11], the consequence of this bias is the appearance of highly coherent pixels inside the changed areas. Within this context, the change detection performance degrades considerably which complicates more the CCD map interpretation particularly when using high resolution SAR data. Medium resolution SAR images obtained 
from satellites such as ERS, Envisat and Alos involve the complex multilooking operation to square the pixels. This operation leads to the improvement of the coherence estimation but at the expense of the spatial resolution. Multilooking is not necessary when using high resolution SAR data such as Cosmo-SkyMed (CSK) and TerraSAR-X since the image pixels are already nearly square. Nevertheless, the speckle is more noticeable in high resolution data and the coherence bias is more important.

In [7] a method is proposed to reduce the bias, based on space-averaging of coherence samples over a local area. This method allows for the enhancement of the probability of detecting changes, but a large window size is still needed to detect the entire changes. The Local Fringe Frequencies (LFF), which is a measure of the interferometric phase variability, is successfully applied in [8] as an additional change indicator to clean the aberrant highly coherent pixels inside changed areas. The use of the LFF to enhance the coherence map permits an important improvement in change detection performance and offers the advantage of preserving the spatial resolution.

The second difficulty of the CCD technique concerns the change identification. As the coherence is affected by several factors (such as baseline decorrelation and volume decorrelation), the coherence map can reveal changes that are not only due to man-made activities [1]. An area of low-backscatter strength (e.g., water surface, smooth surface, shadows) leads to decorrelation in the coherence image which is not truly the change of interest. The identification of the changes remains difficult and further investigation is needed.

In this chapter, a classification scheme is proposed with eight classes and the significance of these classes. The change classification is based on the improved coherence map combined with the pair of SAR intensity images to identify the types of change (man-made activity, natural surface decorrelation,...etc.). The two SAR intensity images are not used to detect changes as in [5], but only to support the coherence map analysis and interpretation. A set of three high resolution CSK SAR images is used, which concerns Goma airport in the Democratic Republic of Congo (DRC). High-resolution visible images are also used in the visual qualitative validation process. The results show that the proposed classification scheme is simple and effective for the change detection and identification, and that it contributes significantly to the overall scene analysis and understanding.

\section{Methods}

\subsection{SAR Interferometry}

InSAR is a technique that exploits the phase differences of at least two SAR images acquired from different orbit positions and/or at different times [3]. In Repeat-pass interferometry the SAR system flies on (ideally) parallel tracks and views the terrain from slightly different directions at different times. Fig. 1 shows a typical spaceborne InSAR configuration. SAR 1 and SAR 2 denote the satellite positions when the first and second SAR images were taken. The distance between them is called the (geometric) baseline and is denoted as $B$ in this figure. The perpendicular baseline, $B_{\perp}$, is the component of $B$ in the direction perpendicular to the SAR 2 look direction. $R_{1}$ and $R_{2}$ denote the ranges to the target $T$ from satellite positions SAR 1 and SAR 2 respectively. The principle of the SAR Interferometry technique 


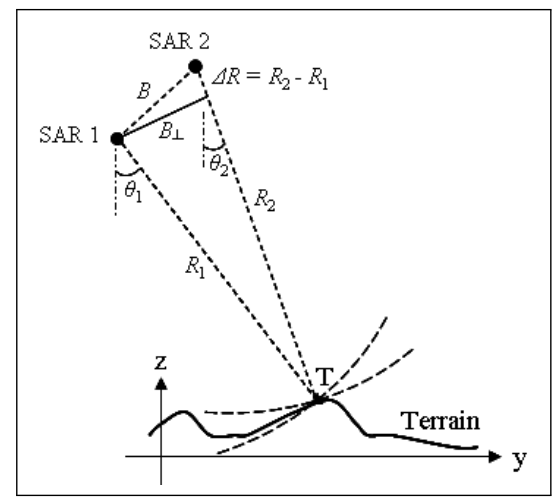

Figure 1. Interferometric configuration. Satellite positions SAR 1 and SAR 2 have ranges, to target $T, R_{1}$ and $R_{2}$ respectively. The separation $B$ between the satellites is called the spatial baseline.

is the use of the phase information of every pixel which is directly related to the parallaxe $\Delta R=R_{2}-R_{1}$. Let

$$
f(R, x)=|f(R, x)| \exp \left\{j \phi_{1}(R, x)\right\} \text { and } g(R, x)=|g(R, x)| \exp \left\{j \phi_{2}(R, x)\right\}
$$

be the two co-registered SAR images forming the interferogram

$$
I(\cdot)=f(\cdot) g^{*}(\cdot)
$$

the phase of which

$$
\phi(\cdot)=\phi_{1}(\cdot)-\phi_{2}(\cdot)
$$

is the interferometric phase.

The phase of the SAR image response $\phi$ of a point scatterer is proportional to range (where $k$ is the factor of proportionality) plus a possible shift $\phi_{\text {scat }}$ due to the scatterer itself [10], i.e.

$$
\begin{aligned}
& \phi_{1}=-2 k R_{1}+\phi_{\text {scat }, 1} \\
& \phi_{2}=-2 k R_{2}+\phi_{\text {scat }, 2}
\end{aligned}
$$

Assuming that there is no possible ground displacement of the scatterers between the observations, the interferometric phase contains the following terms [3]:

$$
\phi=\phi_{\text {topo }}+\Delta \phi_{\text {scat }}
$$

where:

- $\phi_{\text {topo }} \cong \frac{4 \pi}{\lambda} \frac{B_{\perp}}{R \sin \theta} z$ is the topographic term; 
- $\Delta \phi_{\text {scat }}$ represents the influence of any change in scattering behavior. It may be a deterministic phase offset (i.e. change in dielectric constant) or a random phase (i.e. temporal decorrelation).

For Digital Terrain Model extraction, the first term $\phi_{\text {topo }}$ is improved when the other is minimized. In CCD applications, the term $\Delta \phi_{\text {scat }}$ is of interest and its variability is measured by the coherence defined in the next section.

\subsection{InSAR coherence}

\subsubsection{Coherence estimation}

In order to provide some measure of discrimination in the SAR image pair and to accommodate the random noise fluctuations, the InSAR coherence is commonly used. The sample coherence, which is defined as the magnitude of the estimated sample complex cross-correlation coefficient between the SAR image pair, encodes the degree of scene similarity as a value in the range $[0,1][6,7]$ :

$$
\gamma_{N} e^{j \phi^{\prime}}=\frac{\sum_{i=1}^{N} f_{i} g_{i}^{*}}{\sqrt{\sum_{i=1}^{N}\left|f_{i}\right|^{2} \sum_{i=1}^{N}\left|g_{i}\right|^{2}}}
$$

where $\gamma_{N}$ is the sample coherence obtained by $N$ measurements, and $\phi^{\prime}$ is the filtered interferometric phase.

As shown in [1], the coherence is affected by different contributions which are mainly related to:

1. the relative backscatter signal to radar receiver noise ratio $(S N R)$ in the interferometric image pair,

2. the baseline decorrelation that is related to the satellite tracks separation, and

3. the temporal decorrelation caused by changes in the land surface, e.g., man-made objects, vegetation change, ploughing or wind action in desert areas, ... etc. It is the dominant factor in the repeat pass SAR interferometry [4].

In order to assess the suitability of using the InSAR coherence to detect man-made changes, some important results of interferometric SAR processing are presented in the following. Fig. 2 shows CSK intensity images and their coherence image in two different environment types. The white pixels of the intensity images indicate objects that reflect strong energy toward the satellite antenna, whereas the dark pixels indicate surfaces that do not reflect energy toward the satellite. In the coherence image, white pixels represent values of coherence near 1 , while dark pixels represent values near 0 .

In the port area, one can clearly distinguish the sea that is indicated by Fig. 2(a)-1, the dock (quay) in Fig. 2(a)-2, and the vehicles terminal in Fig. 2(a)-3. In the coherence image of Fig. 2(c), the sea area is characterized by low coherence values as it concerns incoherent medium. The coherence of the quay is preserved, as indicated in Fig. 2(c)-2, because of the absence of disturbance in the surface roughness. For the vehicles terminal, the coherence is 


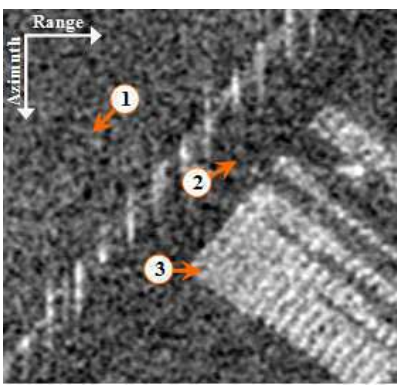

(a)

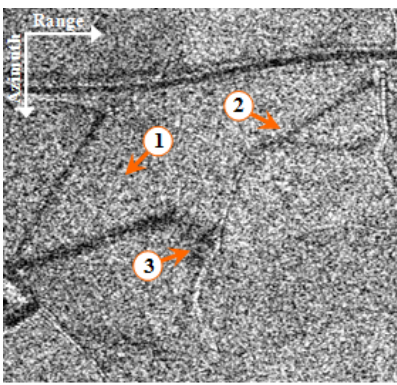

(d)

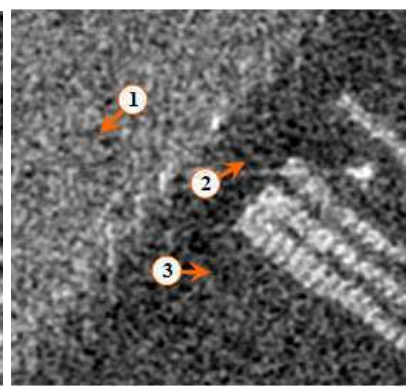

(b)

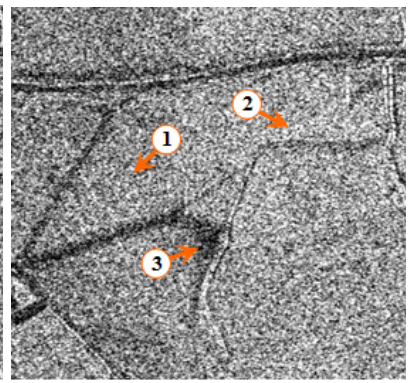

(e)

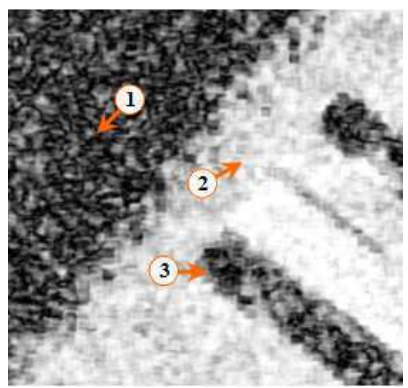

(c)

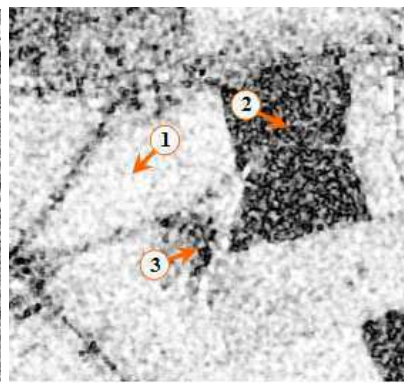

(f)

Figure 2. SAR intensity image pairs and the corresponding coherences. (a) CSK intensity image of June 15, 2011 acquired over a port area. (b) CSK intensity image of June 19, 2011. (c) Coherence image of June 15 and 19, 2011. (d) CSK intensity image of January 1, 2010 acquired over an agricultural area. (e) CSK intensity image of January 9, 2010. (f) Coherence image of January 1 and 9, 2010. Imaged scene size of $300 \times 300 \mathrm{~m}^{2}$.

low in Fig. 2(c)-3, as a result of moved vehicles between the two acquisition dates. The changes related to the man-made objects, such as vehicles and containers, may also be detected via incoherent techniques, which only use SAR intensity images [12]. However, in this situation the coherence can offer the advantage of being able to detect changes even if a vehicle are moved and replaced by another one, which is very similar. This is not possible with incoherent methods.

The use of coherence to measure surface changes is even more interesting for the analysis of examples in agricultural environment. The two intensity images of Fig. 2(d) and Fig. 2(e) are nearly identical showing the limited capability of incoherent methods. With an interferometric SAR processing, the cultivated parcels are clearly indicated by low coherence values (Fig. 2(f)-2), while the uncultivated parcels are characterized by high coherence values (Fig. 2(f)-1). In terms of the interferometric phase, which mainly measures the terrain topography, the obtained values are quite steady in the undisturbed areas, while the values are randomly distributed in the changed areas causing a loss of coherence. In military applications, most of the interesting man-made changes, such as vehicle tracks in a no-man's land, are not indicated by the intensity images but rather by the interferometric phase. This is true in particular for X-band SAR data that are highly sensitive to centimeter changes in the scatterers distribution within resolution cell [4]. 


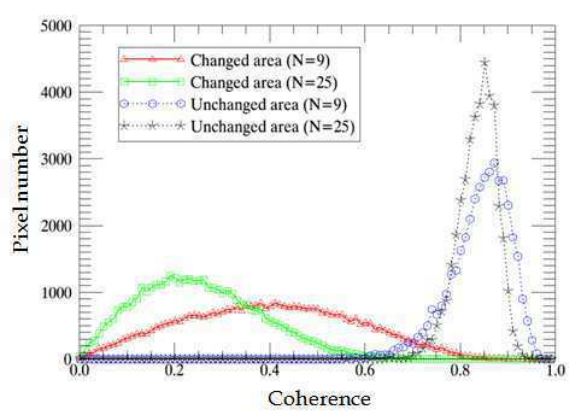

(a)

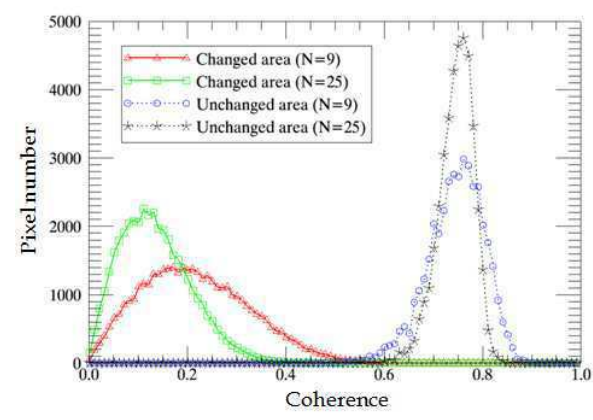

(b)

Figure 3. InSAR coherence histograms of the cultivated and uncultivated areas using different number $N$ of samples. (a) Coherence evaluated without complex multilooking. (b) Coherence evaluated with complex multilooking of 4 pixels.

The results in Fig. 2 demonstrate the appropriateness of the SAR coherence, which uses both intensity images and interferometric phase to detect all man-made changes. However, there are two difficulties to overcome. On the one hand, the coherence can indicate some changes as in Fig. 2(f)-3, which are not of interest but only due to low backscattering (SNR) in the two SAR images (Fig. 2(d)-3 and Fig. 2(e)-3). This situation, that mainly occurs in presence of smooth surfaces (roofs, roads, shadows,...etc.) constitutes the main raison for what we propose in the present work: to develop a classification scheme that permits identification of man-made changes. On the other hand, there is a problem of coherence misestimation, causing highly coherent pixels inside changed areas which complicates the coherence image interpretation.

\subsubsection{Coherence bias}

As demonstrated in [7], the InSAR coherence estimator is biased especially for low coherence values. The bias is due to the limited sample size ( $N$ measurements) in the numerical computation of the Estimate (7). Fig. 3 depicts histograms of coherence images evaluated for different number of samples $N$. It can be seen that the coherence bias induces the presence of highly coherent pixels inside changed areas (light-colored pixels in Fig. 2(f)-2).

The coherence bias decreases when the number of samples used to estimate the coherence increase. For example, the coherence evaluated using $N=5 \times 5$ represents more faithfully the coherence of the changed area than the coherence evaluated by $N=3 \times 3$, as there are less samples of high coherence inside the changed area. According to Fig. 3-(a), the coherence mean value of the cultivated parcel is 0.24 with $N=5 \times 5$ against 0.43 for $N=$ $3 \times 3$. Note that the coherence histograms correspond to the same area of Fig. 2(f)-2, and that the discrepancy in the results is only due to the increasing number $N$ of samples. On the contrary, in case of an undisturbed area as in Fig. 2(f)-1, the coherence mean value is practically not affected by the increase of $N$. According to Fig. 3-(a), the coherence mean value of the unchanged area is 0.82 for both $N=3 \times 3$ and $5 \times 5$, which may be explained by the fact that the coherence estimate is particularly biased for the low coherence values. Fig. 3-(b) shows that the use of the complex multilooking of the SAR images leads to a better 


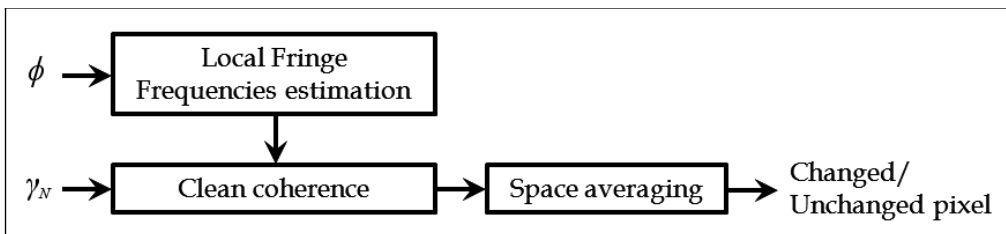

Figure 4. Scheme of the LFF-based CCD method.

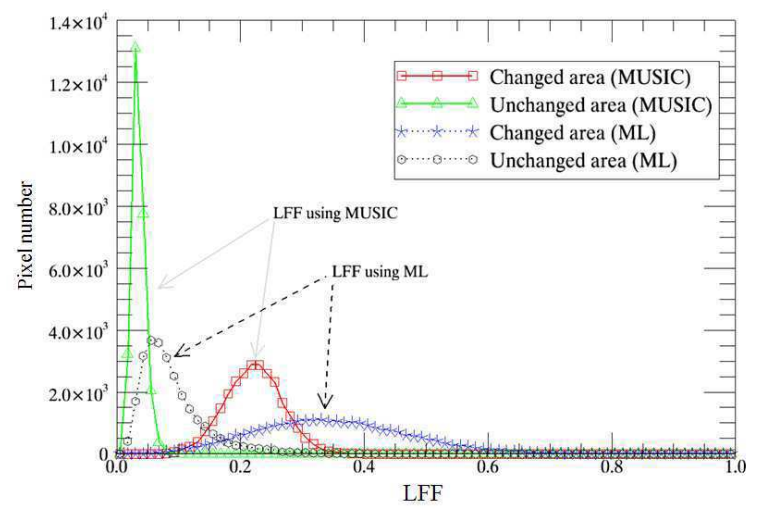

Figure 5. LFF histograms of the cultivated and uncultivated parcels of Figure 2(f).

separation between the changed and unchanged areas, but at the expense of the spatial resolution.

The results of Fig. 3, similar to those in [7], show the necessity to increase the number of samples to obtain a good separation between coherence values of the changed and unchanged areas. In CCD applications, the challenge consists in separating as much as possible the changed and the unchanged pixels, using small window-size $N$ and without multilooking, to preserve subtle changes in the coherence image as much as possible.

In this work, the SAR interferometric processing is done in full resolution with a small window-size of $N=3 \times 3$ pixels, corresponding approximately to an area of $2.4 \times 2.4 \mathrm{~m}^{2}$. According to Fig. 3, this corresponds to the most unfavorable situation of bad separation between the changed and unchanged classes, thus an additional processing step of coherence bias removal is necessary to improve the coherence map.

\subsubsection{Coherence map improvement using LFF}

The basic space averaging operation over $M$-pixel local area is commonly performed for further coherence bias removal [7]:

$$
z=\frac{1}{M} \sum_{i=1}^{M} \gamma_{N i} \underset{H_{1}}{\stackrel{H_{0}}{\gtrless}} T_{C}
$$


where $H_{0}$ is a realization of the null hypothesis (scene changes of interest absent) and $H_{1}$ is the alternative hypothesis (scene changes of interest present). To make the decision, the statistic $z$ is compared to a detection threshold $T_{c}$ [8].

As shown in [8], a large number of samples $N$ and a large window-size $M$ are needed to detect the entire set of changes, which is obviously at the expense of the preservation of small changes. For that reason, another information source must be used to better analyze the coherence image. In the following paragraphs, we describe a method for the coherence map improvement, which is based on the exploitation of the LFF information.

Using a 2-D notation, the complex InSAR phase can be modeled by a first-order approximation (for simplicity, noise is neglected) [13]:

$$
e^{j \phi(k, l)}=e^{j 2 \pi\left(k f_{x}+l f_{y}\right)}
$$

where $f_{x}$ and $f_{y}$ are the LFF components in range and azimuth directions respectively.

According to Equation (6) and by assuming that the neighboring pixels have the almost identical terrain height, which is valid only using high resolution images, the change components may also be obtained by directly differentiating the interferometric phase,

$$
\left\{\begin{array}{l}
\Delta \phi_{\text {scat }-x}=\phi(k+1, l)-\phi(k, l) \\
\Delta \phi_{\text {scat }-y}=\phi(k, l+1)-\phi(k, l)
\end{array}\right.
$$

According to (9) and (10), the LFF estimates may correspond to the change components that can be obtained by differentiating the interferometric phase directly, a process which is not restricted to the first-order model that is due to the fact that $\phi(k, l)$ in (7) includes all the frequencies components $\left(f_{x i}, f_{y i}\right)$ that exist inside the $N$-pixel local area [13]. Therefore, the measured interferometric phase is highly influenced by noise. In this context, the LFF estimation can be obtained via either the Maximum Likelihood (ML) method [14] or the MUltiple SIgnal Classification (MUSIC) method [15].

An analysis of Fig. 5 indicates that the two LFF histograms corresponding to the cultivated and uncultivated parcels of Fig. 2(f) are far apart. This confirms that the LFF components represent an additional indicator of changes. Here, the MUSIC method is used since it provides better LFF histograms separation as shown in Fig. 5. Various SAR image pairs have been used to evaluate LFF histograms for both changed (cultivated parcels, sea area) and unchanged areas. It was observed that the location of the histograms' intersection does not change significantly. As a results, it is possible to set the LFF threshold to $T_{l}=0.1$ as shown in Fig. 5, an important aspect for window-size adaptation.

Here, the coherence improvement is achieved using the LFF information as an additional change indicator, to clean the highly coherent pixels inside the changed areas that are also characterized by high LFF values [8].

Fig. 6 depicts InSAR coherence maps obtained with and without the LFF information. The area of interest concerns fields with a dense agricultural activity. The result of a simple thresholding method (without space-averaging, i.e. $M=1$ ) of the coherence, see Fig. 6(a), is not usable due to the presence of a large number of highly coherent pixels inside the changed 


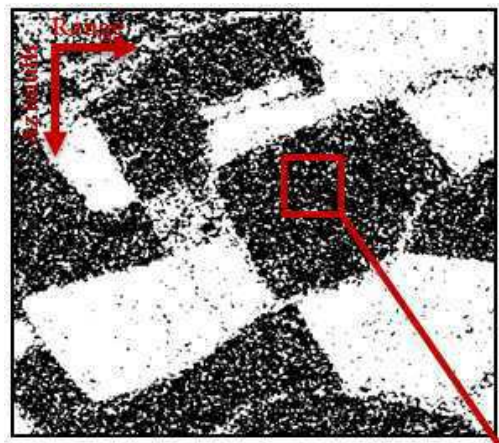

(a)

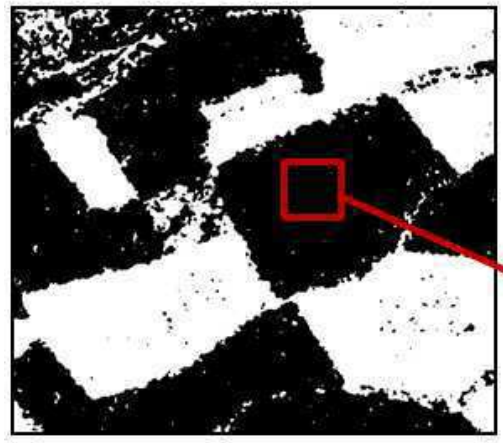

(c)

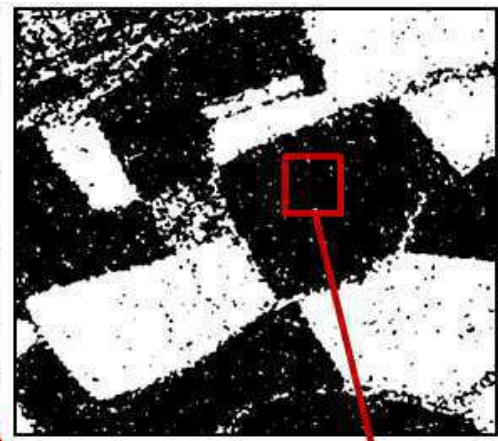

(b)
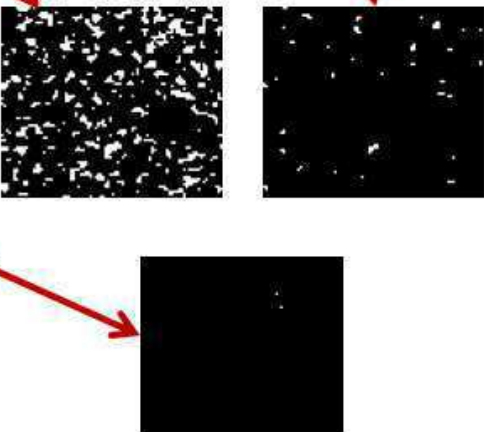

(d)

Figure 6. InSAR coherence result using $N=3 \times 3$ samples. (a) Coherence map without space-averaging $(M=1)$. (b) Coherence map with space-averaging $(M=3 \times 3)$. (c) Change map using the LFF information. (d) Zoom on a cultivated parcel. White pixels indicate unchanged areas, while dark pixels indicate changed areas.

area. In this case, a probability of detection $P_{d}=0.64$ is only achieved, leading to an unusable coherence map. An enhancement of the result is obtained by space-averaging coherence, see Fig. $6(\mathrm{~b})$, using a $3 \times 3$ window. In this case, the detection probability improves to $P_{d}=0.94$ but remains insufficient. The best result is obtained by using the LFF information, as presented in Fig. 6(c), with a detection probability $P_{d}=0.99$. In this case, the highly coherent pixels inside changed areas are mostly reduced (detection of almost all changes). The zoom of the changed area, as presented in Fig. 6(d), clearly indicates that the method using the LFF information outperforms the existing coherence space-averaging method.

\subsection{Proposed classification scheme}

After the InSAR coherence improvement step, the detected changes must be identified as the coherence is affected by several decorrelation sources. To identify the man-made activities in a CCD map, the improved coherence map was combined with the two corresponding SAR intensity images. Fig. 7 presents a schematic overview of the processing chain used for the change classification. 


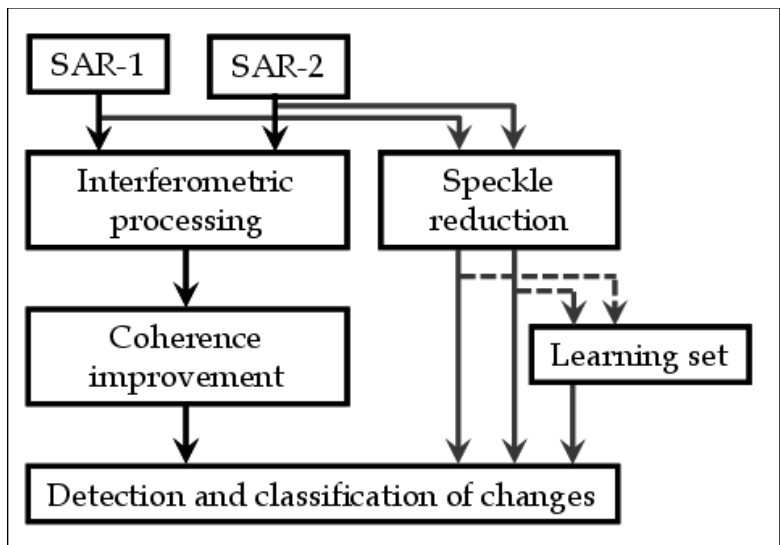

Figure 7. Schematic overview of the change detection and classification.

\begin{tabular}{|c|c|c|c|c|}
\hline \multirow{2}{*}{ Class } & \multicolumn{3}{|c|}{ Features } & \multirow{2}{*}{ Interpretation } \\
\hline & $\begin{array}{l}\ddot{\mathscr{U}} \\
\tilde{0} \\
\frac{0}{0} \\
\frac{0}{0}\end{array}$ & $\underset{\sim}{\stackrel{1}{c}}$ & 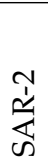 & \\
\hline C1 & $\mathrm{L}$ & $\mathrm{L}$ & $\mathrm{L}$ & $\begin{array}{l}\text { Specular surfaces: water, roads, } \\
\text { roofs, shadows }\end{array}$ \\
\hline C2 & $\mathrm{L}$ & $\mathrm{L}$ & $\mathrm{H}$ & Man-made objects present in SAR-2, not in SAR-1 \\
\hline C3 & $\mathrm{L}$ & $\mathrm{H}$ & $\mathrm{L}$ & Man-made objects present in SAR-1, not in SAR-2 \\
\hline C4 & $\mathrm{L}$ & $\mathrm{H}$ & $\mathrm{H}$ & $\begin{array}{l}\text { Man-made object present in both images but } \\
\text { it changed from SAR-1 to SAR-2 }\end{array}$ \\
\hline C5 & $\mathrm{H}$ & $\mathrm{L}$ & $\mathrm{L}$ & Bare soil or low vegetation \\
\hline C6 & $\mathrm{H}$ & $\mathrm{L}$ & $\mathrm{H}$ & $\begin{array}{l}\text { Invalid class (problem of intensity thresholding } \\
\text { caused by speckle) }\end{array}$ \\
\hline C7 & $\mathrm{H}$ & $\mathrm{H}$ & $\mathrm{L}$ & $\begin{array}{c}\text { Invalid class ( problem of intensity thresholding } \\
\text { caused by speckle) }\end{array}$ \\
\hline C8 & $\mathrm{H}$ & $\mathrm{H}$ & $\mathrm{H}$ & $\begin{array}{l}\text { Scatterers present in both scenes: fixed structures } \\
\text { (e.g. parts of buildings, railways), undisturbed areas }\end{array}$ \\
\hline
\end{tabular}

Table 1. Overview of the eight classes resulting from the change detection and classification.

Human activity is thus characterized by a low coherence and a high intensity in at least one of the two SAR images. However, any decision based on SAR intensity is hampered by the presence of speckle which leads to an increase in the number of false alarms. Therefore, a speckle reduction is performed prior to the change classification: a $3 \times 3$ Lee filter [16] is applied to SAR intensity images in this work. The developed classification scheme is quite simple and is based on a combination of thresholds on the three features, i.e. the enhanced coherence and the two corresponding Lee-filtered intensity images. The coherence map threshold corresponds to the intersection of coherence histograms of the changed (cultivated field) and unchanged areas of Fig. 3 [8].

The intensity threshold is determined from the histograms intersection of the learning sets to sub-divide each of the two SAR images into a low $(\mathrm{L})$ and a high $(\mathrm{H})$ value area. The learning set consists in two classes: low backscattered area (smooth surface or water surface) and 


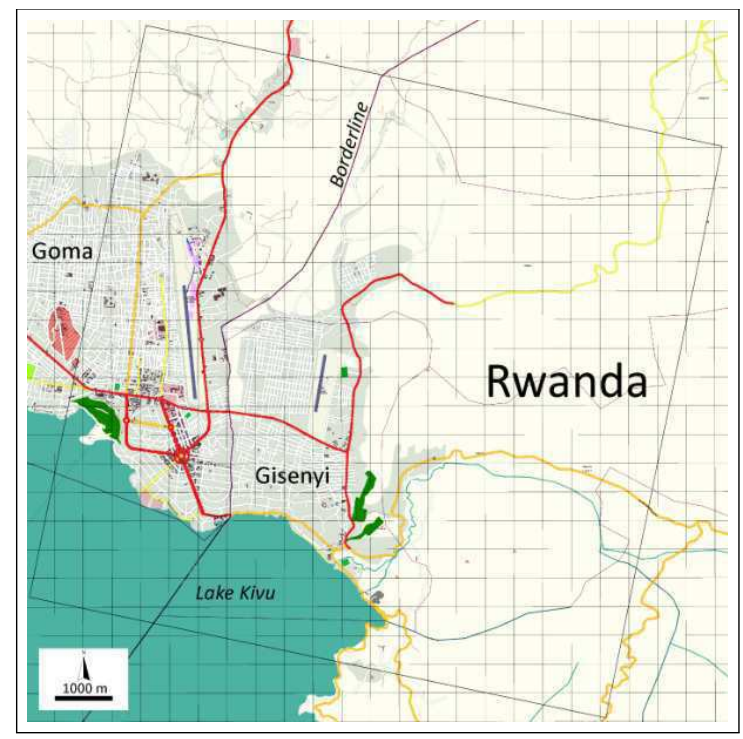

Figure 8. Geographical location of the test site in Goma (DRC) that is characterized by a flat topography. The grey rectangle indicates the borders of the imaged scene corresponding to the used CSK SAR images of $14568 \times 14376$ pixels.

high backscattered area (rough surface). For each of the two classes an area of approximately $200 \times 200$ pixels is identified in the scene. The actual change detection and characterization is a rule-based set of decisions applied to the thresholded SAR images and to the coherence map.

Dividing each of the three feature sets into two value regions leads to eight possible combinations, thus eight possible classes. Table 1 presents an overview of the properties of these classes. Classes of interest for change detection and activity monitoring are $\mathrm{C} 2, \mathrm{C} 3$ and C4. Classes $\mathrm{C} 1, \mathrm{C} 5$ and $\mathrm{C} 8$ contribute to the overall scene understanding. C6 and C7 represent classes in which the coherence is high but the intensity changes between the two images. If this situation occurs, it is most probably due to the fact that the intensity value exceeds the threshold in a region where it should not (the tails of the histograms).

It is also possible to divide the coherence into 3 regions; low $(\mathrm{L})$, medium $(\mathrm{M})$ and high $(\mathrm{H})$. This situation would lead to 12 classes that complicate further the CCD map analysis and interpretation. For this reason a binary thresholding of the coherence had been chosen first, which proves to be sufficient for identification of all man-made changes. In future work, the influence of the intermediate (medium) level on the quality of the final result will be analyzed.

\section{Experimental results}

\subsection{Data and study areas}

The results presented in this work are obtained using the X-band CSK SAR images, horizontally polarized in spot-light mode. The CSK images are acquired on $24 \mathrm{March}, 28$ 
March and 1 April 2011, with an incident angle of $26^{\circ}$. The pixel resolution is $0.73 \mathrm{~m}$ in the ground range direction and $0.7 \mathrm{~m}$ in the azimuth direction. The test site, shown in Fig. 8, concerns the Goma airport in DRC. For the validation of the classification scheme, high-resolution visible images are used with the SAR intensity images to serve as ground truth. The co-registration of the SAR images was performed with Sarscape software (Sarmap 2012). Programs and algorithms were developed in Interactive Data Language (IDL).

The area of interest, shown in Fig. 9(a), concerns a part of the Goma airport that is a busy and flat test site. The airport was under extension, and an important earth moving activity was recorded during the acquisition period of 24 March to 1 April 2011. The scene concerns a wide open field surrounded by buildings and an urban area. Four zones are identified in the test site of Fig. 9(a). Firstly, the area occupied by the company in charge of the airport extension, as indicated by Fig. 9(a)-1, is surrounded by roads where excavators are clearly visible. The anthropic activities are mainly concentrated in the dike that is indicated by Fig. 9(a)-2 and in an area pointed by Fig. 9(a)-3. The dike causes a distortion (shadow) in the SAR image as indicated in Fig. 9(b)-2. The fourth zone concerns the airport runway that is indicated by Fig. 9(a)-4. It is characterized by a low backscattering power as shown in Fig. 9(b)-4. In the urban area, left side of Fig. 9(b), most of the roofs are characterized by a low backscattering power.

\subsection{Results and analysis}

Fig. 9(c) shows the InSAR coherence map of the imaged area obtained using the CSK acquisitions of March 24 and 28, 2011. Light-colored pixels represent values of coherence near 1 , while dark pixels represent values near 0 . Areas with high coherence indicate the absence of surface perturbation. Places with low coherence, corresponding to the areas disturbed between the two acquisitions dates, are located e.g. in Fig. 9(c)-1, 2 and 3. All roads leading to the holding area of Fig. 9(c)-1 have lost the coherence, due to the movements of the excavators.

The analysis and interpretation of the InSAR coherence map become complicated in the presence of man-made structures. Indeed, for example, the big building in Fig. 9(c)-1 is characterized by a low coherence in Fig. 9(c)-1, which is not truly the change that is of interest. The change classification results (or CCD map) corresponding to the period of 24 and 28 March 2011 are depicted in Fig. 9(d). Only the changes C2, C3 and C4 are of interest, and the other classes help to analyze the scene. Note that the incoherent change detection methods can detect the $\mathrm{C} 2$ and $\mathrm{C} 3$ changes, but fail to detect the $\mathrm{C} 4$ changes (type of change that is revealed by the interferometric phase). Besides the advantages of the CCD technique, the classification contributes to the scene analysis. The large building in Fig. 9(d)-1, and most of the urban area on the left side of Fig.9(d), is now classified as C1 instead of change in the coherence map.

The analysis of the results of Fig. 9(c) and Fig. 9(d) shows that in a simple environment without obstacles (i.e., open field in the middle of the scene shown in Fig. 9(a)), the classification method identifies the man-made changes as belonging to classes $\mathrm{C} 2, \mathrm{C} 3$ or C4 while the coherence map confuses the changes in a single category. In a complex environment, e. g. of the urban area in the left part of the scene presented in Fig. 9(a) and the airport runway in Fig. 9(c)-4, the InSAR coherence map becomes hard to interpret and the proposed change classification scheme identifies well the man-made changes and contributes 


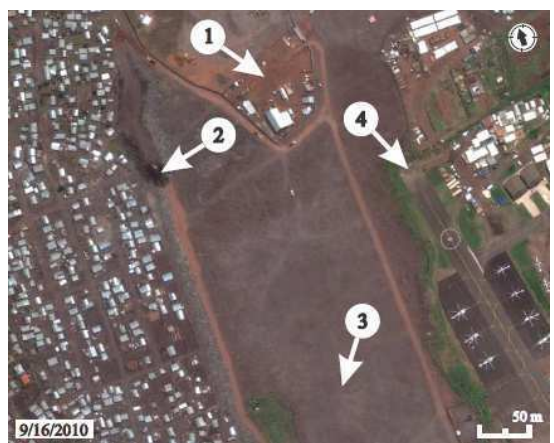

(a)

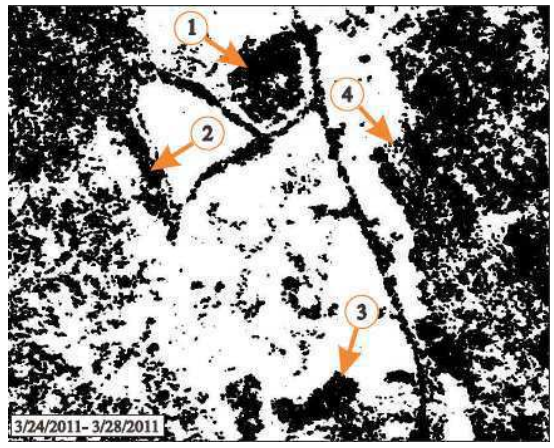

(c)

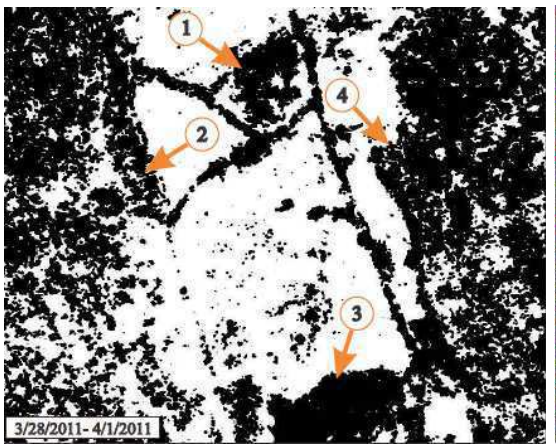

(e)

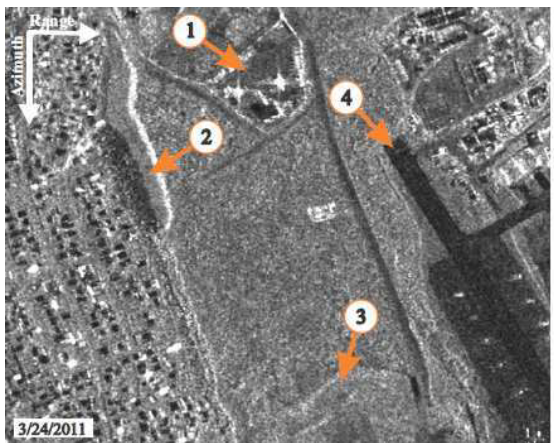

(b)

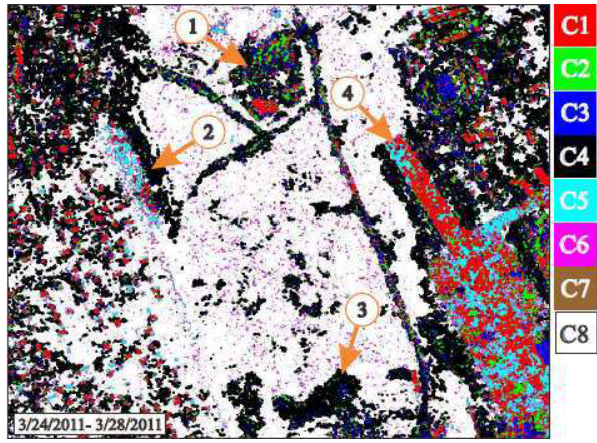

(d)

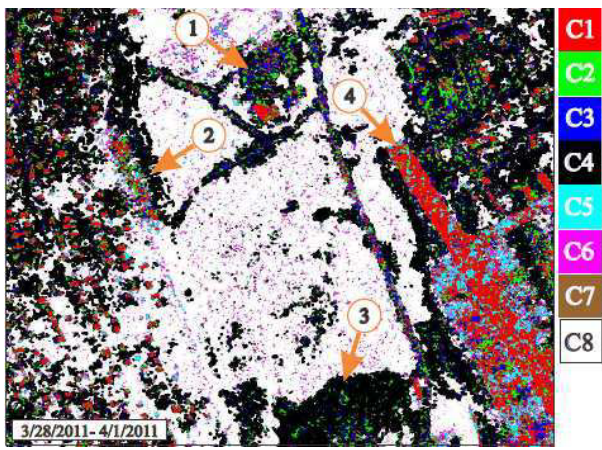

(f)

Figure 9. Change classification results. (a) Visible image of the test site. (b) CSK SAR image intensity of March 24, 2011. (c) Coherence map of March 24 and 28, 2011, normal baseline $=307 \mathrm{~m}$. (d) Change classification results (CCD map) between March 24 and 28, 2011. (e) Coherence map of March 28 and April 1, 2011, normal baseline $=65 \mathrm{~m}$. (f) Change classification results (CCD map) between March 28 and April 1, 2011. COSMO-SkyMed TM Product - ASI [2011] processed under license from ASI - Agenzia Spaziale Italiana. All rights reserved. 
significantly to the overall scene understanding. The results of Fig. 9(e) and Fig. 9(f), obtained using an other image pair (28 March and 1 April 2011) confirm the validity of the classes proposed in Table 1. All areas of the scene are classified in the same way in Fig. 9(d) and Fig. 9(f), except in the presence of man-made changes, as in Fig. 9(d)-3. In addition, the results show that class $\mathrm{C} 5$ is encountered only in the specular surface and shadowed areas; it can be also assimilated to class C1. Despite the complexity of the environment, the invalid classes $\mathrm{C} 6$ and $\mathrm{C} 7$ are rarely present in the analyzed scene. In general, if classes C6 and C7 are significantly present in the classification result, it is an indication that the value of the intensity threshold should be revised.

\section{Conclusion}

This work deals with the development and the validation of a new method of coherent change detection and classification. The InSAR coherence map is enhanced and combined with the two corresponding SAR intensity images to build a CCD map using a simple change classification scheme. The proposed method is tested successfully using high resolution COSMO-SkyMed images. The test area of interest concerns the Goma airport, which is a flat and busy test site.

In a complex environment, the obtained results show that the InSAR coherence map reveals changes but remains hard to interpret. The proposed change classification scheme identifies well the man-made changes, and contributes significantly to the overall scene analysis and understanding. The results obtained using other image pairs confirm the validity of the proposed changed-unchanged classes. The proposed method is an improvement for the analysts in charge of the exploitation of information derived from radar imagery.

In future work, we will analyze the influence of the intermediate thresholding of the coherence on the quality of the final CCD map.

\section{Acknowledgements}

The Authors would like to thank the private companies Sarmap and EXELisvis for their support in providing a full SarscapeTM license (v.4.8, 2011) embedded in the latest version of ENVI software. The COSMO-SkyMed SAR images were obtained through collaboration within the SPARE-SAFE project, launched by the Belgian Ministry of Defense, and processed at the CISS Department of the Royal Military Academy of Belgium.

\section{Author details}

Azzedine Bouaraba ${ }^{1}$, Nada Milisavljević ${ }^{2}$, Marc Acheroy ${ }^{2}$, Damien Closson ${ }^{2}$

1 Radar and Microwave Laboratory, École Militaire Polytechnique, Algiers, Algeria

2 Department of CISS, Royal Military Academy, Brussels, Belgium

\section{References}

[1] H. A. Zebker and J. Villasensor. Decorrelation in interferometric radar echoes. IEEE Trans. and Geosci. Remote Sens., Vol. 30(5):950-959, 1992. 
[2] E. J. M. Rignot and J. J. V. Zyl. Change detection techniques for ers-1 sar data. IEEE Trans. and Geosci. Remote Sens., Vol. 31(4):896-906, 1993.

[3] D. Massonnet and K. L. Feigl. Radar interferometry and its applications to changes in the earth's surface. Review of Geophysics, Vol. 36(4):441-500, 1998.

[4] M. D. Preiss, A. Gray, and N. J. S. Stacy. Detecting scene changes using synthetic aperture radar interferometry. IEEE Trans. Geosci. and Remote Sens., Vol. 44(8):2041-2054, 2006.

[5] X. Boli, M. C. Jing, and K. Gangyao. A change detection measure based on a likelihood ratio and statistical properties of sar intensity images. Remote Sensing Letters, Vol. 3(3):267-275, 2012.

[6] D. G. Corr and A. Rodrigues. Coherent change detection of vehicle movements. In Proceedings of IGARSS, pages 2451-2453, May 1998.

[7] R. Touzi, A. Lopes, J. Bruniquel, and P. W. Vachon. Coherence estimation for sar imagery. IEEE Trans. Geosci.and Remote Sens., Vol. 37(1):135-149, 1999.

[8] A. Bouaraba, D. Borghys, A. Belhadj-Aissa, M. Acheroy, and D. Closson. Improving ccd performance by the use of local fringe frequencies. Progress In Electromagnetics Research C, Vol. 32:123-137, 2012.

[9] N. Oishi. A change detection measure based on a likelihood ratio and statistical properties of sar intensity images. In Proceedings of the 6th European Radar Conference, pages 278-281, Rome, Italy, October 2009.

[10] D. Just and R. Bamler. Phase statistics of interferograms with applications to synthetic aperture radar. Applied Optics, Vol. 33(20):4361-4368, 1994.

[11] C. L. Martinez. Coherence estimation in synthetic aperture radar data based on speckle noise modeling. Applied Optics, Vol. 46(4):544-558, 2007.

[12] J. Im, J. R. Jensen, and J. A. Tullis. Object based change detection using correlation image analysis and image segmentation. Remote Sensing Letters, Vol. 29(2):399-423, 2008.

[13] Z. Suo, Z. Li, and Z. Bao. A new strategy to estimate local fringe frequencies for insar phase noise reduction. IEEE Geosci. Remote Sens. Lett., Vol. 40(7):771-775, 2010.

[14] U. Spagnolini. 2-d phase unwrapping and instantaneous frequency estimation. IEEE Trans. Geosci. Remote Sens., Vol. 33(5):579-589, 1995.

[15] E. Trouvé, M. Caramma, and H. Maitre. Fringe detection in noisy complex interferograms. Applied Optics, Vol. 35(20):3799-3806, 1996.

[16] J. S. Lee. Digital image enhancement and noise filtering by use of local statistics. IEEE Trans. on Pattern Analysis and Machine Intelligence, Vol. 2:165-168, 1980. 
